\title{
RENAL AUTOTRANSPLANTATION IN A PATIENT WITH ACUTE RENAL INFARCTION AFTER SURGERY FOR A DISSECTING ANEURYSM
}

Nobushige Tamura, MD, ${ }^{\mathrm{a}}$ Kwansong Ku, MD, PhD, ${ }^{\mathrm{a}}$ Yasumasa Shichiri, MD, ${ }^{\mathrm{b}}$ Yoshiya Sakurai, MD, ${ }^{\mathrm{a}}$ Masanori Nishimura, MD, ${ }^{\mathrm{b}}$ Rikiya Shioyama, MD, ${ }^{\mathrm{b}}$ Morihiro Kondoh, MD, ${ }^{\mathrm{c}}$ Kazunobu Nishimura, MD, PhD, and Masashi Komeda, $\mathrm{MD}, \mathrm{PhD},{ }^{\mathrm{d}}$ Kyoto, Japan

Reduced perfusion of abdominal organs may occur in patients with dissection of the thoracic and abdominal aorta. ${ }^{1}$ We report a case involving a 58-year-old woman with acute renal failure who had thrombosed infarction after an operation for a DeBakey type I dissection. The patient presented with severe lumbar pain and exacerbated renal function caused by acute renal arterial thrombosis. Renal autotransplantation after renal arterial thrombectomy produced a normalization of blood flow to the diseased kidney and a gradual recovery of renal function.

Clinical summary. A 58-year-old woman had a sudden onset of severe chest and back pain and visited our center in October 1999. Contrast-enhanced computed tomography (CT) revealed a DeBakey type I, Stanford type A, acute aortic dissection. The CT also demonstrated a 50-mm ascending aorta and dissection from the ascending aorta through the abdominal aorta to the level of the left renal artery. A cardiopulmonary bypass (CPB) procedure was performed with the use of 2 venous cannulas and a femoral arterial return. The perioperative transesophageal echocardiogram showed an intimal tear in the ascending aorta without valvular abnormality. Therefore, we performed graft replacement of the ascending aorta. After establishment of CPB, retrograde blood cardioplegic arrest at $22^{\circ} \mathrm{C}$ in combination with circulatory arrest was carried out. The aneurysm was resected and replaced with a woven doublevelour graft (Hemashield; Meadox Medicals, Inc, Oakland, NJ [subsidiary of Boston Scientific Corporation, Natick, Mass]). The patient was weaned from CPB unaided, with normal hemodynamic indexes. On the first postoperative day, she had oliguria and showed a sudden rise in serum creatinine and blood urea nitrogen (BUN) levels to $5.1 \mathrm{mg} / \mathrm{dL}$ and 70.2 $\mathrm{mg} / \mathrm{dL}$, respectively, necessitating hemodialysis. She required daily hemodialysis or hemofiltration for 20 days. Thereafter, renal function recovered (serum creatinine, $3.2 \mathrm{mg} / \mathrm{dL}$; serum BUN, $33.3 \mathrm{mg} / \mathrm{dL}$ ), and dialysis was no longer performed. However, on postoperative day 26, the patient had sudden lumbar pain. Unheralded oliguria was associated with worsening renal function (serum creatinine, $5.0 \mathrm{mg} / \mathrm{dL}$; serum BUN, 44.0 $\mathrm{mg} / \mathrm{dL}$; creatinine clearance, $8.6 \mathrm{~L} / \mathrm{d}$ ).

From the Departments of Cardiovascular Surgery, ${ }^{\mathrm{a}}$ Urology, ${ }^{\mathrm{b}}$ and Nephrology, ${ }^{\mathrm{c}}$ Otowa Hospital, and the Department of Cardiovascular Surgery, ${ }^{\mathrm{d}}$ Graduate School of Medicine, Kyoto University, Kyoto, Japan.

J Thorac Cardiovasc Surg 2001;121;985-6

Copyright () 2001 by The American Association for Thoracic Surgery

$0022-5223 / 2001 \$ 35.00+0 \quad \mathbf{1 2 / 5 4 / 1 1 0 6 8 0}$

doi:10.1067/mtc.2001.110680
A CT scan at this point revealed infarction of the left kidney (Fig 1). During the operation, the left kidney was excised for heterotopic autotransplantation. Extensive thrombosis within a true lumen of the left renal artery was revealed. After removal of the thrombus and perfusion with heparinized cold saline solution, renal autotransplantation to a heterotopic site in the pelvis with the left mobilized internal iliac artery for end-to-end anastomosis to the distal renal artery and the left common iliac vein for side-to-end anastomosis to the renal vein was performed. The patient required hemodialysis for 5 days. Thereafter, renal function recovered gradually. On postoperative day 118 (second operation), serum creatinine and BUN levels were 0.8 and $9.6 \mathrm{mg} / \mathrm{dL}$, respectively, and creatinine clearance was $52.7 \mathrm{~L} / \mathrm{d}$. The postoperative magnetic resonance imaging scan showed a functioning autotransplanted kidney (Fig 2). She was discharged 5 months later.

Discussion. Renal ischemia is a widely known cause of renal failure that results from a dissecting aortic aneurysm extending to the level of the renal arteries. In our patient, after surgical treatment of DeBakey type I dissection, a risk of renal function was suspected as a result of renal infarction by arterial thrombosis. After thrombectomy of the left renal artery, heterotopic renal autotransplantation was subsequently performed, and the patient's renal function normalized. Renal autotransplantation is the procedure of choice for reperfusion of an ischemic kidney complicated by renal arterial stenosis or occlusion with renovascular hypertension. ${ }^{2}$ Renal autotransplantation has been performed in the treatment of renal hypertension in patients with Takayasu's arteritis. ${ }^{3}$

Thomson and associates ${ }^{4}$ reported good results with this technique in the treatment of acute renal failure after a progression of a dissection beyond the origin of the renal arteries in Marfan syndrome. This is the first report of successful renal autotransplantation in a patient with acute renal failure caused by left renal arterial thrombosis after surgical treatment of a DeBakey type I dissection. In the present patient the left renal artery was occluded as a result of extensive thrombosis, which occasionally occurs after obliteration of the true lumen. Although we cannot explain the reason why the left renal artery was occluded by a clot, stasis of blood flow might cause thrombosis in the true lumen that has been compressed and narrowed by the false one, or perhaps some thrombi of unknown origin might obstruct the true lumen of the left renal artery. In addition, we were afraid that the dissection may also extend to the right renal artery that branches off of the smaller true lumen, but the CT scan revealed a somewhat functioning right kidney, and thus we elected not to perform bilateral autotransplantation and followed the progress of the right kid- 


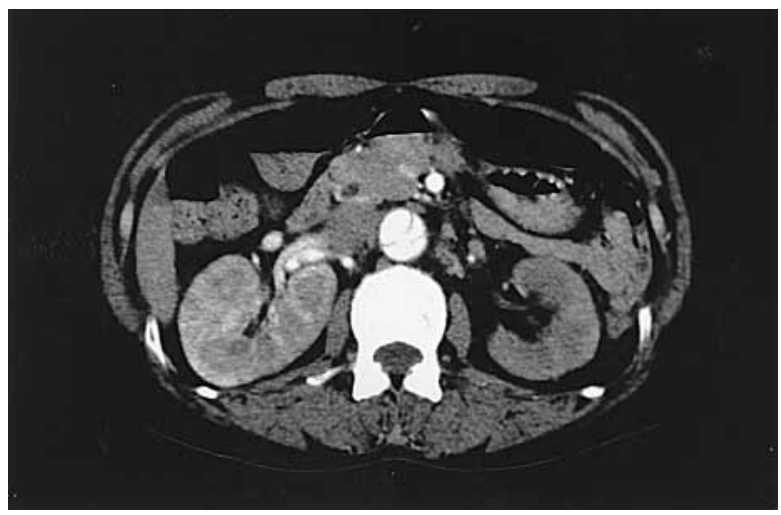

Fig 1. Contrast-enhanced CT scan showing infarction of the left kidney.

ney. Slonim and associates ${ }^{5}$ reported that percutaneous balloon fenestration of the intimal flap and endovascular stenting is an effective treatment for life-threatening ischemic complications of acute aortic dissection. We considered flap fenestration to treat left renal ischemia; however, the ultrasonic echoangiogram that was performed just before the second operation showed no blood flow in a true lumen of the left renal artery that was not collapsed or narrowed by the false lumen. We therefore considered that left renal ischemia occurred not because of the occlusion by an intimal flap but because of the left renal arterial thrombosis. Indeed, during the operation, extensive thrombosis within the left renal artery was revealed, and we successfully performed the left renal autotransplantation to a heterotopic site in the pelvis after removal of the thrombus. In our experience it appears that heterotopic renal autotransplantation by which normal arterial perfusion distal to the dissection is re-established is a good therapeutic option for reperfusion of the ischemic kidney compromised by a progressive dissection of the thoracoabdominal aorta.

Received for publication May 23, 2000; accepted for publication Aug 1, 2000.

Address for reprints: Kwansong $\mathrm{Ku}, \mathrm{MD}$, Department of Cardiovascular Surgery, Otowa Hospital, 2 Chinji-cho, Yamashina-ku, Kyoto 607-8062, Japan.

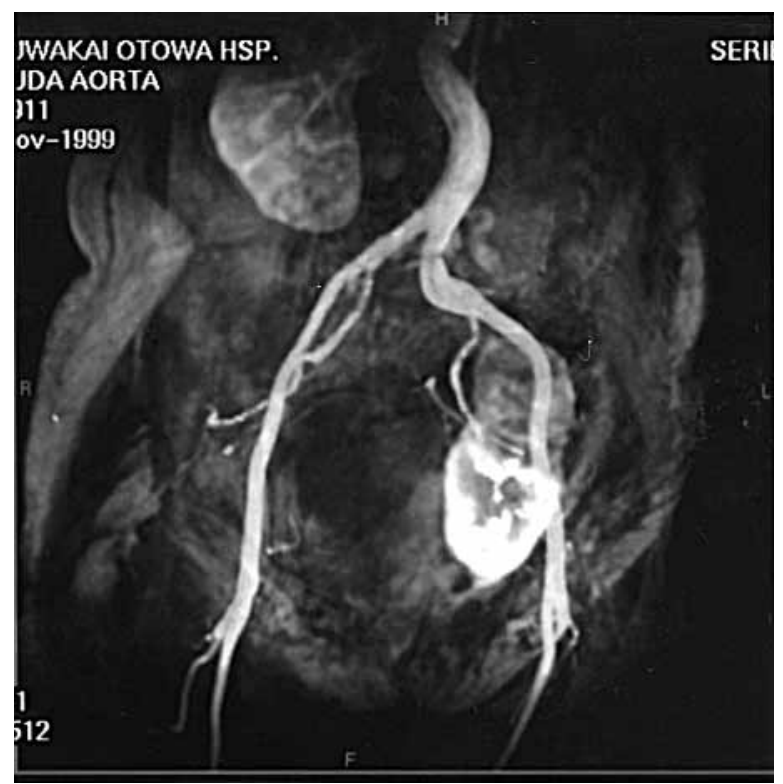

Fig 2. Postoperative magnetic resonance imaging scan confirming a functioning autotransplanted kidney.

\section{REFERENCES}

1. O'Neil EA, Hansen KJ, Canzanello VJ, Pennell TC, Dean RH. Prevalence of ischemic nephropathy in patients with renal insufficiency. Am J Surg 1992;58:485-90.

2. Beale PG, Meyers KEC, Thomson PD. Management of renal hypertension in children with Takayasu's arteritis using renal autografting or allograft transplantation in selected circumstances and total lymphoid irradiation. J Pediatr Surg 1992;27:836-9.

3. Kyriakides GK, Najarian JS. Renovascular hypertension in childhood: successful treatment by renal autotransplantation. Surgery 1979;83:611-6.

4. Thomson SD, Chmura AM, Slapak M. Renal autotransplantation for aortic dissection in Marfan's syndrome. J R Soc Med 1995;88:532-3.

5. Slonim SM, Miller DC, Mitchell RS, Semba CP, Razavi MK, Dake MD. Percutaneous balloon fenestration and stenting for life-threatening ischemic complications in patients with acute aortic dissection. J Thorac Cardiovasc Surg 1999;117:1118-27.

\section{Targeted}

The Journal of Thoracic and Cardiovascular Surgery gives you two tables of contents.

The condensed table of contents tells you at a glance what topics and authors are presented each month. The expanded table of contents gives you a brief abstract of each article. You select only those articles of most interest to you for further reading. 